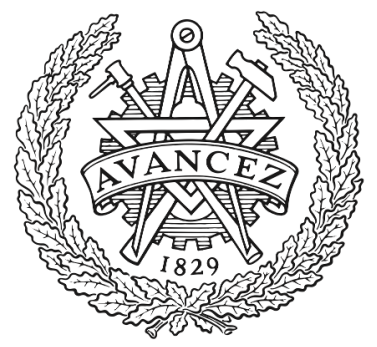

CHALMERS

UNIVERSITY OF TECHNOLOGY

\title{
Adjacent Channel Interference Aware Joint Scheduling and Power Control for V2V Broadcast Communication
}

Downloaded from: https://research.chalmers.se, 2023-04-26 09:44 UTC

Citation for the original published paper (version of record):

Unnichiriyath Siddique, A., Yuan, D., Ström, E. et al (2021). Adjacent Channel Interference Aware Joint Scheduling and Power Control for V2V Broadcast Communication. IEEE Transactions on Intelligent Transportation Systems, 22(1): 443-456. http://dx.doi.org/10.1109/TITS.2020.2968526

N.B. When citing this work, cite the original published paper.

(C)2021 IEEE. Personal use of this material is permitted.

However, permission to reprint/republish this material for advertising or promotional purposes 


\title{
Adjacent Channel Interference Aware Joint Scheduling and Power Control for V2V Broadcast Communication
}

\author{
Anver Hisham, Di Yuan, Senior Member, IEEE, Erik G. Ström, Senior Member, IEEE, \\ and Fredrik Brännström, Member, IEEE
}

\begin{abstract}
This paper proposes scheduling and power control schemes to mitigate the impact of both co-channel interference (CCI) and adjacent channel interference (ACI) on direct vehicleto-vehicle broadcast communication. The objective is to maximize the number of vehicles that can communicate with the prescribed requirement on latency and reliability. The joint scheduling and power control problem is formulated as a mixed Boolean linear programming (MBLP) problem. A column generation method is proposed to reduce the computational complexity of the joint problem. From the joint problem, we formulate a schedulingalone problem (given a power allocation) as a Boolean linear programming (BLP) problem and a power control-alone problem (given a schedule) as an MBLP problem. The scheduling problem is numerically sensitive due to the high dynamic range of channel values and adjacent channel interference ratio (ACIR) values. Therefore, a novel sensitivity reduction technique, which can compute a numerically stable optimal solution at the price of increased computational complexity, is proposed. Numerical results show that ACI, just as CCI, is a serious problem in direct vehicle-to-vehicle (V2V) communication due to near-far situations and hence should not be ignored, and its impact can be reduced by proper scheduling and power control.
\end{abstract}

\section{INTRODUCTION}

\section{A. Motivation}

$\mathrm{V} 2 \mathrm{~V}$ communication can reduce traffic accidents significantly by broadcasting up-to-date local and emergency informations to nearby vehicles. To this end, both periodic and event-driven messages are conveyed. Periodic messages are transmitted by all vehicular user equipments (VUEs) in order to convey its current status to neighbors such as position, velocity and acceleration, whereas event-driven messages are sent when any emergency situation has been detected. However, conveying such safety related messages requires the establishment of highly reliable, low latency broadcast communication links between VUEs.

Anver Hisham, Erik G. Ström, and Fredrik Brännström are with the Communication Systems Group, Department of Electrical Engineering, Chalmers University of Technology, SE-412 96 Gothenburg, Sweden. E-mail: \{anver erik.strom, fredrik.brannstrom\}@ chalmers.se

Di Yuan is with the Department of Science and Technology, Linköping University, 60174 Norrköping, Sweden. E-mail: di.yuan@liu.se

This work has been performed in the framework of the H2020 project 5GCAR co-funded by the EU. The authors would like to acknowledge the contributions of their colleagues. The views expressed are those of the authors and do not necessarily represent the project. The simulations were performed in part on resources provided by the Swedish National Infrastructure for Computing (SNIC).

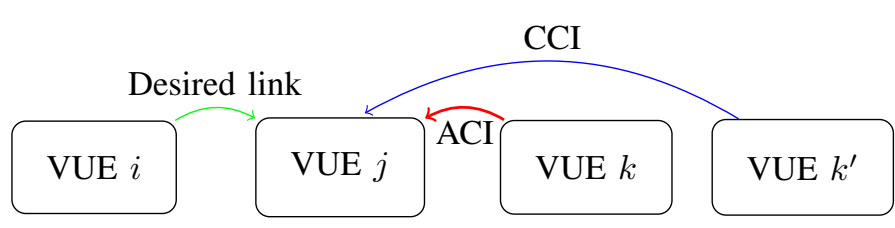

Fig. 1: System model

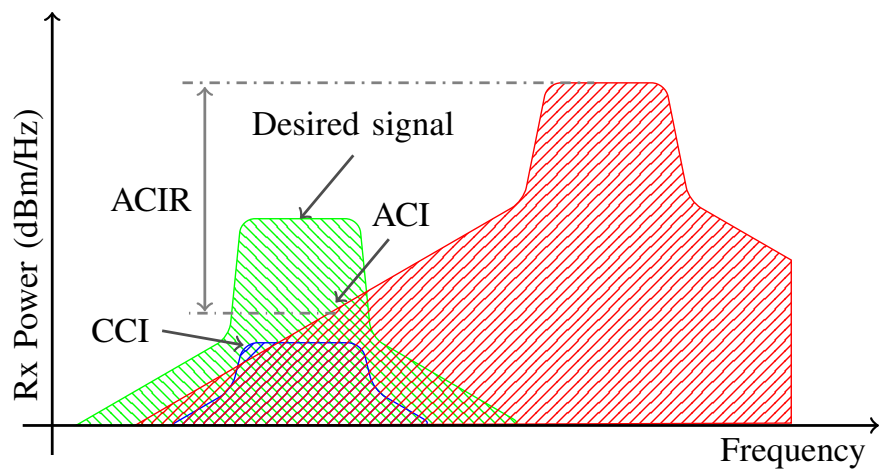

Fig. 2: Received power spectral density at receiving VUE $j$.

In this context, 3GPP is standardizing both cooperative awareness messages (CAMs) for periodic messages, and decentralized environmental notification messages (DENMs) for aperiodic messages in V2X communication. CAMs are sent with a frequency of $2-10 \mathrm{~Hz}$, and the latency requirement is $100 \mathrm{~ms}$, depending upon the application [1, Table 1]. DENMs are used to alert vehicles of a detected event, and the transmission can be repeated and persist as long as the event is present [2]. However, both periodic and aperiodic messages in $\mathrm{V} 2 \mathrm{~V}$ are local and broadcast in nature, requiring cooperation between vehicles in close proximity.

The reliability requirement of $\mathrm{V} 2 \mathrm{~V}$ links can be achieved by ensuring that the signal to interference and noise power ratio (SINR) is above a certain threshold value [3]. The SINR of a wireless link depends on the received interference power. Broadly speaking, there are two types of interferences: cochannel interference (CCI) and adjacent channel interference (ACI). CCI is cross-talk between transmitters scheduled in 
same time-frequency slot, whereas ACI is interference due to leakage of transmit power outside the intended frequency slot. Hence, ACI will affect transmissions that are scheduled in same timeslot but at different frequency slots.

ACI is mainly caused by the nonlinearity of the power amplifier (PA) in the transmitter. Advanced methods have been developed to linearize the PA [4]-[7], however, the clipping effect of the PA cannot be avoided, which results in ACI. An example of ACI is illustrated in Figs. 1-2, where VUE $i$ is transmitting to VUE $j$, and VUEs $k$ and $k^{\prime}$ are interfering. The interfering VUE $k$ is transmitting on a nearby frequency slot causing ACI, whereas, VUE $k^{\prime}$ is transmitting on the same frequency slot as desired slot, therby causing CCI. However, as illustrated in the figure, when the receiver VUE $j$ is closer to VUE $k$ than VUE $k^{\prime}$, the ACI caused by VUE $k$ is larger than the CCI caused by VUE $k^{\prime}$. Therefore, the received signal to interference and noise ratio (SINR) of VUE $j$ is mostly limited by the ACI from VUE $k$ rather than the CCI from VUE $k^{\prime}$, even though VUE $k$ is transmitting on an adjacent frequency slot.

ACI is typically measured using a parameter named adjacent channel interference ratio (ACIR), which is defined as the ratio of received signal power in the transmitted frequency slot to the received ACI in the nearby frequency slot. In other words, ACIR is the ratio between the average in-band received power from transmitter $k$ and the average out of band received power from transmitter $k$ 's signal in the frequency slot allocated for transmitter $i$, as illustrated in Fig. 2.

The performance of a communication network is limited by ACI when 1) VUEs are multiplexed in frequency domain, and 2) a near-far scenario occurs. If the number of available timeslots is not enough for scheduling each VUE individually in each timeslot, then the interference includes ACI. The ACI becomes a determining factor in communication when nearfar situations occur, i.e., when the desired signal is weak compared to ACI as shown in Fig. 2. The near-far situations are more common in direct $\mathrm{V} 2 \mathrm{~V}$ broadcast communication since (i) the transmit powers tend to be similar in broadcast communication, (ii) all transmitted signals are of interest to all receivers, and (iii) the obstruction by intermediate vehicles cause high penetration loss [8]-[11] resulting in high dynamic range in channel gains. This implies that the received power ratio from a nearby and far-away transmitter is high, especially when there are many blocking vehicles, and ACI can therefore be a significant problem when detecting the far-away signal. Therefore, ACI should not be ignored when designing scheduling and power control for direct V2V broadcast communication.

\section{B. State of the Art}

There are many studies on scheduling for V2V communication that consider reliability and latency requirements [12][14]. Out-of-cellular coverage scenario for V2V is theoretically studied in [15], and a simulation-based study is found in [16]. Infrastructure-based scheduling is studied in [17], while a distributed autonomous scheduling approach is proposed in
[18]. V2V scheduling algorithms for broadcast service are studied in [18], [19]. Location-based scheduling for a highway platooning scenario is investigated in [20], [21]. In [22], the authors study scheduling strategies in the absence of channel state information (CSI). A strategy for joint optimization of scheduling and power control for sidelink (which includes $\mathrm{V} 2 \mathrm{~V}$ ) is presented in [23]. To improve connectivity among VUEs, a scheduling strategy based on inter-VUE distances and mobility is proposed in [24], which can reduce the access delay. In [25], a joint scheduling and power control algorithm is proposed for $\mathrm{V} 2 \mathrm{~V}$ networks with the objective to satisfy certain delay aware quality of service $(\mathrm{QoS})$ requirements, under the assumption of instantaneous CSI knowledge. In the same paper, the authors prove that the optimal power allocation scheme in theory converges to the classical water-filling policy with the considered objective. A study on maximum achievable V2V datarates has been done in [26], where a network coding technique to cancel the interference is proposed. The authors also consider road side units (RSU) for the information exchange. A study on the capability of the IEEE 802.11p standard to satisfy stringent $\mathrm{V} 2 \mathrm{~V}$ requirements is done in [27], [28], where the authors conclude that the IEEE 802.11p standard may not be sufficient. Instead, the authors propose a novel medium access control (MAC) protocol and transport layer for IEEE 802.11p in order to satisfy real-time V2V requirements. However, most of the existing literature focuses on reducing $\mathrm{CCI}$ alone, without considering ACI. But ACI is a significant factor in $\mathrm{V} 2 \mathrm{~V}$ communication due to nearfar problem, hence affect the performance [29]. In [30], the authors analyze the impact of ACI for device-to-device (D2D) communication, for various user densities and transmit power, and conclude that ACI indeed causes outage when the user density is high. Extensive studies have been done to measure the impact of ACI when different communication technologies coexist in adjacent frequency bands [31]-[34]. In [35], the authors assess the performance degradation due to ACI when two LTE base stations are deployed in adjacent frequency channels. The impact of ACI on $802.11 \mathrm{~b} / \mathrm{g} / \mathrm{n} / \mathrm{ac}$ was also been broadly studied [36]-[38].

The impact of ACI on V2V carrier-sense multiple access (CSMA) MAC layer has been studied in [39], [40]. The authors conclude that a potential transmitter would falsely assume that the channel is busy due to the ACI from a transmitter tuned to an adjacent channel, which causes the transmitter to defer its transmission resulting in delays.

We note that adequate attention has not yet been given to study the effect of ACI within a V2V broadcast communication scenario. This scenario, in which each VUE is simultaneously a transmitter and a receiver, is not mainstream, and it is hard to find related scheduling and power control work in the existing literature. Additionally, CCI-based scheduling and power control may not help to mitigate the impact of ACI [41], and does not generalize well to account for ACI. Our previous works [29], [41] try to understand the impact of ACI upon vehicular communication, and mitigate it using scheduling and power control algorithms. In this paper, we 
generalize our previous work in two main directions by 1) allowing overlapping scheduling for all VUEs in the network and thereby enabling completely general reuse of timefrequency resources, 2) reducing the computational complexity and numerical sensitivity for joint scheduling and power control.

\section{Contributions}

Our goal is to maximize the number of connected VUEs in a vehicular network, using proper scheduling and power control schemes. We make the following contributions in this paper:

1) The joint scheduling and power control problem to maximize the number of connected VUEs is formulated for general reuse of time-frequency resources, as a mixed Boolean linear programming (MBLP) problem.

2) In order to reduce the computational complexity with respect to the number of timeslots, a novel column generation method is proposed for the joint scheduling and power control problem.

3) The scheduling for fixed power is formulated as a Boolean linear programming (BLP) problem, and power control for a fixed schedule is formulated as an MBLP problem. Both formulations allow for general reuse of time-frequency resources.

4) A cutting plane method is proposed for the scheduling problem to completely remove the numerical sensitivity due to the high dynamic range of channel values and ACIR values.

\section{PRELIMINARIES}

\section{A. Notation}

We use the following notation throughout the paper. Sets are denoted by calligraphic letters, e.g., $\mathcal{X}$, with $|\mathcal{X}|$ denoting its cardinality, and $\emptyset$ indicating an empty set. Lowercase and uppercase letters, e.g., $x$ and $X$, represent scalars. Lowercase boldface letters, e.g., $\mathbf{x}$, represent a vector where $x_{i}$ is the $i^{\text {th }}$ element. The uppercase boldface letters, e.g., $\mathbf{X}$, denote matrices where $X_{i, j}$ indicates the $(i, j)^{\text {th }}$ element. The notation $\mathbb{1}\{$ statement $\}$ is either 1 or 0 , depending upon if the statement is true or false. If there are multiple statements in $\mathbb{1}\{\cdot\}$, the notation is true if and only if all the statements are true.

\section{B. Assumptions}

We have following assumptions;

1) We define $\mathcal{N}=\{1,2, \ldots, N\}$ as the set of VUEs, $\mathcal{F}=\{1,2, \ldots, F\}$ as the set of frequency slots, and $\mathcal{T}=\{1,2, \ldots, T\}$ as the set of timeslots for scheduling. A frequency slot $f$ in timeslot $t$ is denoted as a resource block (RB) $(f, t)$. The framework in this paper does not assume a particular RB size. However, in the numerical results presented in Sec. VI we adopt the LTE RB dimensions, i.e., $180 \mathrm{kHz} \times 0.5 \mathrm{~ms}$ [42, section 5.2.3].

2) A VUE $i \in \mathcal{N}$ wants to broadcast its packet to the VUEs in the set $\mathcal{R}_{i} \subset \mathcal{N}$. For convenience, we define the set of intended transmitters for receiver $\operatorname{VUE} j$ as $\mathcal{T}_{j} \triangleq\{i: j \in$
TABLE I: Key Mathematical Symbols

\begin{tabular}{cl}
\hline Symbol & Definition \\
\hline$N$ & Number of VUEs \\
$F$ & Number of frequency slots \\
$T$ & Number of timeslots \\
$\gamma^{T}$ & SINR threshold to declare a link as successful \\
$\sigma^{2}$ & Noise power in an RB \\
$P^{\text {max }}$ & Maximum transmit power of a VUE \\
$P_{i, f, t}$ & Transmit power of VUE $i$ in an RB in timeslot $t$ \\
$H_{i, j}$ & Average channel power gain from VUE $i$ to VUE $j$ \\
$\lambda_{r}$ & ACI from any frequency slot $f$ to frequency slot $f \pm r$ \\
$X_{i, f, t}$ & Indicate if VUE $i$ is scheduled to transmit in RB $(f, t)$ \\
$\Gamma_{i, j, f, t}$ & SINR of the link $(i, j)$ in RB $(f, t)$ \\
$Y_{i, j, f, t}$ & Indicate if link $(i, j)$ is successful in RB $(f, t)$ \\
$Z_{i, j}$ & Indicate if link $(i, j)$ is successful in any RB \\
$V_{i, k, r, t}$ & Indicate if VUE $i$ and $k$ are scheduled no more than \\
& $r$ frequency slots apart in timeslot $t$ \\
\hline
\end{tabular}

$\mathcal{R}_{i}$. We note that $\mathcal{T}_{j}, j \in \mathcal{N}$ is completely determined by $\mathcal{R}_{i}, i \in \mathcal{N}$ and vice versa. As an example, the set $\mathcal{R}_{i}$ could be all vehicles within a certain distance from VUE $i$; however, the proposed method does not rely on any particular structure for $\mathcal{R}_{i}$ or, therefore, $\mathcal{T}_{j}$. Moreover, we define the set $\mathcal{L}=\left\{(i, j): i \in \mathcal{N}, j \in \mathcal{R}_{i}\right\}$ as the set of all intended links.

3) A centralized controller exists which can schedule and power control all VUEs in the network in $\mathcal{F} \times \mathcal{T}$ RBs. A base station (BS), or a RSU, or a VUE can act as a centralized controller. It is worth mentioning that $3 \mathrm{GPP}$ proposes installing intelligent transport system stations (ITS-S) at regular distances on the roadside, which can relay messages or act as a centralized controller [2].

4) The large-scale channel parameters (i.e., pathloss and penetration loss) are assumed to be slowly varying compared to the scheduling interval $T$. Therefore, we assume that the centralized controller has access to the slowly varying CSI between all pairs of VUEs to compute the average SINR. If this assumption is violated, e.g., if the CSI is missing, noisy or outdated, then optimal scheduling and power control might not be possible or performance will be degraded.

5) A VUE can successfully transmit a message in an RB (i.e., transmit with a message error probability below a predetermined threshold), if the received average SINR is above a certain threshold $\gamma^{\mathrm{T}}[3$, Lemma 1]. The maximum transmit power of a VUE is assumed to be $P^{\max }$.

\section{System Model}

Key mathematical symbols are listed in Table I. We indicate a transmitting VUE as VUE $i$, receiving $\operatorname{VUE}$ as $\operatorname{VUE} j$, and interfering VUE as VUE $k$, as illustrated in Fig. 1. Similarly the link $(i, j)$ indicates the link from VUE $i$ to $\operatorname{VUE} j$. The parameter $H_{i, j}$ is the average channel power gain from VUE $i$ to VUE $j$. Hence, $H_{i, j}$ takes into account pathloss, penetration loss, and large-scale fading between VUE $i$ and VUE $j$. 
Assume that VUE $i$ is transmitting in RB $(f, t)$ and VUE $k$ in $\mathrm{RB}\left(f^{\prime}, t\right)$. If interferer VUE $k$ is transmitting on the same RB as VUE $i$ (i.e., $f^{\prime}=f$ ), then the VUE $j$ 's reception of the transmission from VUE $i$ is affected by CCI from VUE $k$. On the other hand, if VUE $k$ is transmitting on a nearby frequency slot of VUE $i$ (i.e., $f^{\prime} \neq f$ ), then the reception is affected by ACI instead.

\section{Problem Formulation}

\section{A. Joint Scheduling and Power Control Problem}

Let $\mathbf{X} \in\{0,1\}^{N \times F \times T}$ be the scheduling matrix defined as follows,

$$
X_{i, f, t} \triangleq\left\{\begin{array}{ll}
1, & \text { if VUE } i \text { is scheduled in RB }(f, t) \\
0, & \text { otherwise }
\end{array} .\right.
$$

Similarly, $\mathbf{P} \in \mathbb{R}^{N \times F \times T}$ is the power matrix, where $P_{i, f, t}$ is the transmit power of VUE $i$ in $\mathrm{RB}(f, t)$. The variable $P_{i, f, t}$ is constrained by the maximum transmit power $P^{\max }$ of a VUE in a timeslot as follows,

$$
\sum_{f=1}^{F} P_{i, f, t} \leq P^{\max } \quad \forall i, t
$$

Moreover, $P_{i, f, t}$ is also constrained by scheduling as follows,

$$
0 \leq P_{i, f, t} \leq P^{\max } X_{i, f, t} \quad \forall i, f, t
$$

Let us consider a link $(i, j)$ in $\mathrm{RB}(f, t)$, i.e., the link from VUE $i$ to VUE $j$ in frequency slot $f$ and timeslot $t$. The total signal power $S_{i, j, f, t}$ and interference power $I_{i, j, f, t}$ received by VUE $j$ while decoding the signal from VUE $i$ in RB $(f, t)$ can be computed as

$$
\begin{aligned}
S_{i, j, f, t} & =P_{i, f, t} H_{i, j}, \\
I_{i, j, f, t} & =\sum_{f^{\prime}=1}^{F} \sum_{\substack{k=1 \\
k \neq i}}^{N} \lambda_{\left|f^{\prime}-f\right|} P_{k, f^{\prime}, t} H_{k, j},
\end{aligned}
$$

where $\lambda_{r}$ is the ACIR from a frequency slot $f$ to frequency slot $f \pm r$. Therefore, $\lambda_{\left|f^{\prime}-f\right|}$ is the ACIR from frequency slot $f^{\prime}$ to $f$. Note that when $f^{\prime}=f$, the interference is CCI, instead of ACI. Therefore, in order to accommodate CCI, we make $\lambda_{0}=1$.

Following (4) and (5), we can compute the SINR $\Gamma_{i, j, f, t}$ of the link $(i, j)$ in $\mathrm{RB}(f, t)$, as follows,

$$
\Gamma_{i, j, f, t}=\frac{S_{i, j, f, t}}{\sigma^{2}+I_{i, j, f, t}},
$$

where $\sigma^{2}$ is the noise power in an RB.

For the link to be successful, the SINR must be above a certain threshold $\gamma^{\mathrm{T}}$, i.e., $\Gamma_{i, j, f, t} \geq \gamma^{\mathrm{T}}$ or

$$
S_{i, j, f, t}-\gamma^{\mathrm{T}} I_{i, j, f, t} \geq \gamma^{\mathrm{T}} \sigma^{2} .
$$

However, it might not be possible to fulfill this condition for all links $(i, j)$ in all RBs $(f, t)$. To select which combinations of $i, j, f$, and $t$ to enforce this condition, we introduce the Boolean matrix $\mathbf{Y} \in\{0,1\}^{N \times N \times F \times T}$, where

$$
Y_{i, j, f, t} \triangleq \begin{cases}1, & \text { if }(7) \text { is enforced } \\ 0, & \text { otherwise }\end{cases}
$$

We can combine (7) and (8) into a single constraint,

$$
S_{i, j, f, t}-\gamma^{\mathrm{T}} I_{i, j, f, t} \geq \gamma^{\mathrm{T}} \sigma^{2}-\eta\left(1-Y_{i, j, f, t}\right)
$$

where $\eta$ is a sufficiently large number to make (9) hold whenever $Y_{i, j, f, t}=0$, regardless of the schedule and power allocation. It is not hard to show that $\eta=\gamma^{\mathrm{T}}\left(N P^{\max }+\sigma^{2}\right)$ is sufficient.

Observe that if $Y_{i, j, f, t}=1$, then the link $(i, j)$ is successful in $\operatorname{RB}(f, t)$ as per (9). Let us define $\mathbf{Z} \in\{0,1\}^{N \times N}$ as successful link matrix, where each element $Z_{i, j}$ indicates the success of link $(i, j)$ in any $\mathrm{RB}(f, t)$, i.e.,

$$
\begin{aligned}
Z_{i, j} & \triangleq \begin{cases}1, & \text { if link }(i, j) \text { is successful }, \\
0, & \text { otherwise },\end{cases} \\
& =\min \left\{1, \sum_{t=1}^{T} \sum_{f=1}^{F} Y_{i, j, f, t}\right\},
\end{aligned}
$$

where the minimum in (10b) is required to not to count successful links between VUE $i$ and VUE $j$ more than once. The objective is to maximize the total number of successful links in $\mathcal{L}$, i.e., maximize $\sum_{(i, j) \in \mathcal{L}} Z_{i, j}$.

We can translate (10b) into the following set of linear constraints,

$$
\begin{aligned}
& Z_{i, j} \geq Y_{i, j, f, t} \\
& Z_{i, j} \leq \sum_{t=1}^{T} \sum_{f=1}^{F} Y_{i, j, f, t} \\
& Z_{i, j} \in\{0,1\}
\end{aligned}
$$

We note that constraint (11a) is redundant, since we do not require a lower bound for $Z_{i, j}$ while maximizing $\sum_{(i, j) \in \mathcal{L}} Z_{i, j}$. Additionally, the last Boolean constraint (11c) can be relaxed to the constraint $Z_{i, j} \leq 1$, since we are trying to maximize $Z_{i, j}$ and $\mathbf{Y}$ is a Boolean matrix.

Putting everything together, we arrive at the following 
MBLP problem for joint scheduling and power control,

$$
\max _{\mathbf{P}, \mathbf{X}, \mathbf{Y}, \mathbf{Z}} \sum_{(i, j) \in \mathcal{L}} Z_{i, j}
$$

s.t.

$$
\begin{aligned}
& P_{i, f, t} H_{i, j}-\gamma^{\mathrm{T}} \sum_{\substack{k=1 \\
k \neq i}}^{N} \sum_{f^{\prime}=1}^{F} \lambda_{\left|f^{\prime}-f\right|} P_{k, f^{\prime}, t} H_{k, j} \\
& \geq \gamma^{\mathrm{T}} \sigma^{2}-\gamma^{\mathrm{T}}\left(N P^{\max }+\sigma^{2}\right)\left(1-Y_{i, j, f, t}\right) \quad \forall i, j, f, t
\end{aligned}
$$

$$
\begin{aligned}
& \sum_{f=1}^{F} P_{i, f, t} \leq P^{\max } \\
& 0 \leq P_{i, f, t} \leq P^{\max } X_{i, f, t} \\
& Z_{i, j} \leq \sum_{t=1}^{T} \sum_{f=1}^{F} Y_{i, j, f, t} \\
& Z_{i, j} \leq 1 \\
& \mathbf{X} \in\{0,1\}^{N \times F \times T} \\
& \mathbf{Y} \in\{0,1\}^{N \times N \times F \times T} \\
& \mathbf{P} \in \mathbb{R}^{N \times F \times T}
\end{aligned}
$$

We can formulate a number of variations of the above problem:

(i) We observe that the variable scheduling matrix $\mathbf{X}$ is redundant for joint scheduling and power control problem formulation (12), hence, can be avoided. By replacing (12d) with the constraint $0 \leq P_{i, f, t} \leq P^{\max }$, we can optimize over power values $\mathbf{P}$ in (12a) and simply compute $X_{i, f, t}$ as $X_{i, f, t}=\mathbb{1}\left\{P_{i, f, t}>0\right\}$ later on. In other words, we are doing only the power control and schedule VUEs in RB $(f, t)$ whenever their transmit powers in the $\mathrm{RB}$ are nonzero. This way we can reduce the number of Boolean variables, hence, make the problem formulation more compact.

(ii) The problem formulation (12) can be translated into a scheduling alone problem by changing the constraint (12d) to

$$
P_{i, f, t}=\bar{P}_{i, t} X_{i, f, t} \quad \forall i, f, t
$$

where $\bar{P}_{i, t}$ is the transmit power of VUE $i$ in timeslot $t$, which is a known value. Thus, the maximization in (12a) is over $\mathbf{X}, \mathbf{Y}$, and $\mathbf{Z}$. The resulting problem is a Boolean linear programming (BLP) problem.

(iii) The problem formulation (12) can be translated into a power control alone problem for an arbitrary scheduling matrix $\mathbf{X}$. That is, we fix the scheduling matrix $\mathbf{X}$ and optimize over $\mathbf{P}, \mathbf{Y}$, and $\mathbf{Z}$ in (12a). Once we know the schedule, we do not need the constraint $(12 b)$ for all VUEs and for all RBs (i.e., $\forall i, j, f, t$ ), instead, we can limit this constraint only for the scheduled RBs for each VUE. The resulting problem is an MBLP problem.

(iv) The problem formulation (12) can be translated into a problem to maximize the minimum number of successful links for a VUE, instead of total number of successful links. In this way, we are guaranteeing at least $L^{*}$ successful links for any VUE. This is done by changing the objective function (12a) as follows,

$$
L^{*}=\max _{\mathbf{P}, \mathbf{X}, \mathbf{Y}, \mathbf{Z}} L
$$

and adding an extra constraint to (12) as follows,

$$
\sum_{j \in \mathcal{R}_{i}} Z_{i, j} \geq L \quad \forall i
$$

(v) To reduce the total power consumption without compromising the number of connected VUEs, the problem formulation (12) objective can be modified as follows,

$$
\max _{\mathbf{P}, \mathbf{X}, \mathbf{Y}, \mathbf{Z}} \sum_{(i, j) \in \mathcal{L}} Z_{i, j}-\beta \sum_{i=1}^{N} \sum_{f=1}^{F} \sum_{t=1}^{T} P_{i, f, t},
$$

where $\beta$ is the weight of the total power consumption in the objective. Upon setting $\beta \leq 1 /\left(N F T P^{\max }\right)$, the sum power minimization does not affect our primary objective of maximizing the connectivity.

(vi) The problem formulation (12) allows for full-duplex communication, i.e., a VUE can simultaneously transmit and receive. Half-duplex communication can be enforced by adding the constraint,

$$
Y_{i, j, f, t} \leq\left(1-X_{j, f^{\prime}, t}\right) \quad \forall i, j, f, f^{\prime}, t
$$

(vii) We can enforce the scheduling to be nonoverlapping, i.e., scheduling at most one VUE in an RB, by adding the following constraint. This way, CCI can be avoided.

$$
\sum_{i=1}^{N} X_{i, f, t} \leq 1 \quad \forall f, t
$$

(viii) A VUE can be limited to transmit in at most one RB per timeslot by adding the constraint

$$
\sum_{f=1}^{F} X_{i, f, t} \leq 1 \quad \forall i, t
$$

This helps in applying sensitivity reduction techniques without much loss in performance, as we will see in Section V.

\section{JOInt SCHEduling ANd POWER CONTROL Using Column Generation Method}

\section{A. Motivation}

Typical self-driving algorithms require high computational capability and large storage [43], and vehicles would be equipped with powerful processing units and memory [44] in near future. Hence, it might be possible for a VUE acting as a centralized controller and doing optimal scheduling and power control by solving (12). However, we observe that the computational complexity of (12) can be reduced by a column generation method inspired by [45], as proposed in this section. 
The worst-case computational complexity of (12) is $\mathcal{O}\left(2^{(N+1) N F T}\left(N^{2}+N F T\right)^{3} / \log \left(N^{2}+N F T\right)\right)$. The factor $2^{(N+1) N F T}$ is for fixing the $(N+1) N F T$ Boolean variables, and the factor $\left(N^{2}+N F T\right)^{3} / \log \left(N^{2}+N F T\right)$ is for solving each of the resulting linear programming (LP) problem using an interior point method [46]. However, with the column generation method proposed in this section, the computational complexity can be reduced to $\mathcal{O}\left(2^{(N+1) N F} T\left(N^{2}+\right.\right.$ $N F)^{3} / \log \left(N^{2}+N F\right)$ ), i.e., the computational complexity is made to linear (instead of exponential) with respect to the number of timeslots $T$. We also observe significant reduction in simulation time while using the column generation method ${ }^{1}$.

A summary of the computational complexity for the considered algorithms can be found in Section VI, Table III.

\section{B. Algorithm}

First, we explain the intuition behind the algorithm. Suppose we have an ordered set of $Q$ distinct single-timeslot power matrices $\tilde{\mathcal{P}}=\left\{\tilde{\mathbf{P}}^{1}, \tilde{\mathbf{P}}^{2}, \ldots, \tilde{\mathbf{P}}^{Q}\right\}$, where $\tilde{\mathbf{P}}^{q} \in \mathbb{R}^{N \times F}, \quad 1 \leq$ $q \leq Q$, can be thought of as the power allocation in a single timeslot. Let $\tilde{\mathbf{Z}}^{q} \in\{0,1\}^{N \times N}$ be the corresponding successful link matrix, i.e., $\tilde{\mathbf{Z}}^{q}$ is the solution to (12) when $T=1$ and $\mathbf{P}$ is fixed and equals to $\tilde{\mathbf{P}}^{q}$. Let $\tilde{\mathcal{Z}}=\left\{\tilde{\mathbf{Z}}^{1}, \tilde{\mathbf{Z}}^{2}, \ldots, \tilde{\mathbf{Z}}^{Q}\right\}$.

Now suppose $Q \geq T$ and that we are constrained to use the power matrices in $\tilde{\mathcal{P}}$ to form a power allocation for $T$ timeslots. That is, we need to choose $T$ matrices $^{2}$ from $\tilde{\mathcal{P}}$, and we define $\mathbf{w} \in\{0,1\}^{Q}$ s.t. $\sum_{q} w_{q}=T$, as the choice indicator vector, i.e., if $w_{q}=1$, then $\tilde{\mathbf{P}}^{q}$ is chosen. The best choice is found by solving the following problem:

$$
\max _{\mathbf{Z}^{\prime}, \mathbf{w}} \sum_{(i, j) \in \mathcal{L}} Z_{i, j}^{\prime}
$$

s.t.

$$
\begin{aligned}
& \sum_{q=1}^{Q} \tilde{Z}_{i, j}^{q} w_{q} \geq Z_{i, j}^{\prime} \quad \forall i, j \\
& \sum_{q=1}^{Q} w_{q} \leq T \\
& Z_{i, j}^{\prime} \in\{0,1\} \quad \forall i, j \\
& w_{q} \in\{0,1\} \quad \forall q
\end{aligned}
$$

Loosely speaking, as $Q$ grows large, we obtain better and better power allocations, and in the limit when $Q \rightarrow \infty$, the solution to (20) will be essentially optimal. Needless to say, this is not a practical approach. Even for a bounded $Q$, the problem (20) is an NP-hard problem, since it is equivalent to a maximum coverage problem [47]. To overcome these practical

\footnotetext{
${ }^{1}$ As an example, the average simulation time was reduced by $63.6 \%$ for $N=20, F=20, T=2$.

${ }^{2}$ Without loss of generality, we restrict the choice to $T$ distinct matrices in $\tilde{\mathcal{P}}$, as choosing the same matrix multiple times cannot increase the objective (12a).

${ }^{3}$ This constraint is equivalent to $\sum_{q=1}^{Q} w_{q}=T$ in the problem formulation. However, formulating the constraint in this way speeds up the solver.
}

difficulties, we propose a method to find a good set of $Q$ single-timeslot power matrices, and then applying a greedy choosing method (with complexity $\mathcal{O}(T)$ ) to approximately solve (20).

Inspired by the column generation method, we split the problem into three separate problems: 1) master problem [M], 2) subproblem $[\mathrm{S}], 3)$ choosing problem [C]. First we iterate between $[\mathrm{M}]$ and $[\mathrm{S}]$, where in iteration $Q$ we generate $\tilde{\mathbf{P}}^{Q}$ and $\tilde{\mathbf{Z}}^{Q}$ to augment $\tilde{\mathcal{P}}$ and $\tilde{\mathcal{Z}}$ respectively. We terminate the iterations once a termination criteria is met, then we apply [C] to choose best $T$ matrices out of $\tilde{\mathcal{Z}}$. The algorithm is illustrated in Algorithm 1 and explained below,

1) Master problem [M]: The problem formulation (20) is modified by relaxing the Boolean constraints (20d-e) to the constraints $0 \leq Z_{i, j}^{\prime} \leq 1,0 \leq w_{q} \leq 1, \forall i, j, q$. The relaxed problem, which we call [M], is an LP problem and therefore easy to solve. The dual values $\Pi_{i, j}$ for each of the constraints (20b) and the dual value $\pi$ for the constraint (20c) are computed and passed on to [S].

2) Subproblem [S]: The problem formulation (12) is modified by setting $T=1$, and the objective (12a) is changed to $\max \sum_{(i, j) \in \mathcal{L}} \Pi_{i, j} Z_{i, j}-\pi$. The modified problem is solved and the solution power matrix is saved as $\tilde{\mathbf{P}}^{Q}$, and successful link matrix as $\tilde{\mathbf{Z}}^{Q}$, where $Q$ is the iteration count. The sets $\tilde{\mathcal{P}}$ and $\tilde{\mathcal{Z}}$ are augmented by adding $\tilde{\mathbf{P}}^{Q}$ and $\tilde{\mathbf{Z}}^{Q}$ respectively. The augmented $\tilde{\mathcal{Z}}$ is passed to the master problem $[\mathrm{M}]$ in the next iteration.

3) Choosing problem $[\mathrm{C}]$ : We choose $T$ power matrices sequentially for timeslots $t=1,2, \ldots, T$ in a greedy manner. That is, for a timeslot $t=t^{\prime}$, we choose the power matrix $\tilde{\mathbf{P}}^{q^{*}} \in \tilde{\mathcal{P}}$ that adds the highest number of successful links, given the power matrix selections already done in the previous timeslots $t=1,2, \ldots, t^{\prime}-1$, as illustrated in Algorithm 1, line 7-12.

Intuitively, in an $[\mathrm{M}]-[\mathrm{S}]$ iteration, the dual values $\Pi_{i, j}$ computed by $[\mathrm{M}]$ would be larger for a recurrently failing link $(i, j) \in \mathcal{L}$ in the previous iterations. Hence, $[\mathrm{S}]$ will prioritize those links in the subsequent iterations. By the theory of linear programming, we stop the iterations once the subproblem objective value becomes zero or negative, which indicate that the master problem cannot improve the solution anymore by augmenting the set $\tilde{\mathcal{P}}$. To control complexity, we limit the number of iterations to at most $C T$, i.e, $Q \leq C T$. For $C=10$ and the scenarios in Section VI, we observe that this bound was never reached. That is, in all instances, $Q<C T$.

After the $[\mathrm{M}]-[\mathrm{S}]$ iteration has terminated, we find the $T$ timeslot power matrix $\mathbf{P}$ by solving the choosing problem $[\mathrm{C}]$. The power matrix $\mathbf{P}$ then yields the scheduling matrix $\mathbf{X}$ as $X_{i, f, t}=\mathbb{1}\left\{P_{i, f, t}>0\right\} \quad \forall i, f, t$.

It should be noted that the column generation method presented in Algorithm 1 might not provide an optimal solution, since 1) $[\mathrm{M}]$ is a relaxed problem formulation of the original problem (20) and 2) [C] is not guaranteed to compute an optimal power matrix selection.

The scalability of the proposed column generation method 


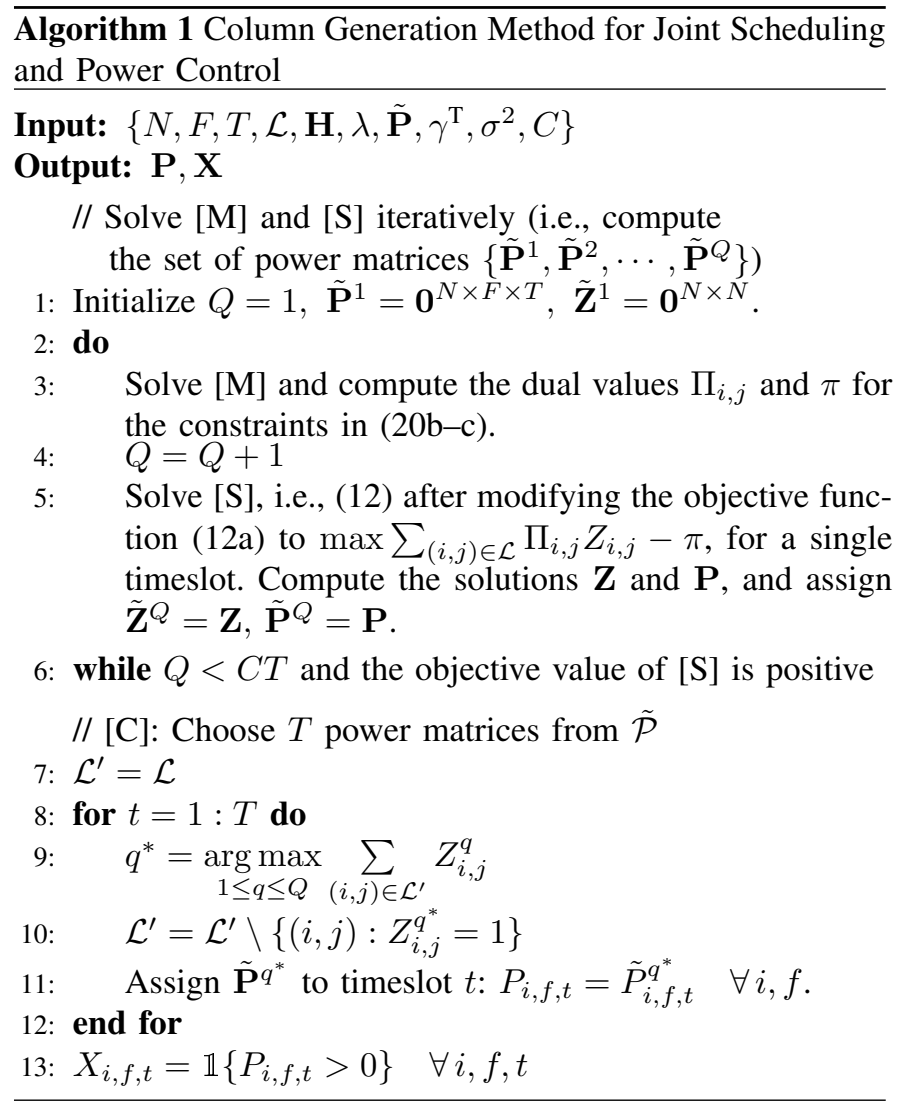

with respect to $N$ is an issue, since high computational complexity of the method forbid solving for larger networks. One solution approach for a large network would be to split the network into smaller networks in a soft manner (to avoid the edge effect), and apply the scheduling and power control algorithms in each small network individually. We omit the details here, and keep the improvement and evaluation of this method as our future work.

\section{Reducing Sensitivity of Scheduling Problem}

In this section, we propose a method to reduce the sensitivity of the scheduling-only version of problem (12), i.e., (12) variant (ii) in Section III. We note that, both ACIR values and $\mathrm{V} 2 \mathrm{~V}$ channel values have got high dynamic rage, which results in both large and small coefficients in the SINR constraint (12b). This makes the constraint (12b) sensitive and the problem (12) numerically harder to solve. Moreover, high numerical sensitivity of the problem leads solver claiming certain links to be successful, which are actually failed links in reality. This leads solver to return a suboptimal solution instead of the optimal solution. To overcome this sensitivity issue, we propose a novel cutting plane approach inspired by [48], which can compute the optimal solution by augmenting (12b) with more robust Boolean cover inequalities. However the proposed method in its current format has higher computational complexity than solving the scheduling problem (12) variant (ii). Therefore, we present this method as a proof of concept, and used mainly for quantifying the optimality gap resulting from the sensitivity of the problem.

The main idea is as follows. We start by solving (12) and find out the violated SINR constraints $(12 b)$ by the resulting solution from the solver. Any resulting SINR violations are identified and used to construct robust and nonsensitive constraints (i.e., cutting planes), which are later added to the scheduling problem. The updated problem is solved, which results in new SINR violations that are used to construct additional robust SINR constraints. The process of iteratively adding robust SINR constraints is repeated until there are no remaining SINR constraint violations. At this point, we have arrived to a feasible solution for the problem (12) which is also optimal (assuming that the modified problem is solved optimally). Optimality follows since the added robust constraints are not stronger than the original constraints (12b).

\section{A. Preliminaries}

In this subsection, we define the variables required for the implementation of the sensitivity reduction method. Let $\bar{I}_{i, j}$ denote the maximum tolerable interference power for a link $(i, j)$. That is, if the received interference power is less than or equals to $\bar{I}_{i, j}$, then the link $(i, j)$ is successful, otherwise, the link is a failure. The value of $\bar{I}_{i, j}$ can be computed from (7) as follows,

$$
\bar{I}_{i, j}=\frac{\bar{P}_{i, t} H_{i, j}}{\gamma^{\mathrm{T}}}-\sigma^{2}
$$

where $\bar{P}_{i, t}$ is the transmit power of VUE $i$ if scheduled in timeslot $t$. We assume $\bar{P}_{i, t}$ is a known quantity since we are considering only scheduling here. A zero value, i.e., $\bar{P}_{i, t}=0$, indicate that VUE $i$ cannot be scheduled in timeslot $t$.

For convenience, we add the constraint (19), which prevents scheduling a VUE to more than one RB per timeslot. The potential loss in performance due to this is probably minimal, since scheduling in multiple RBs in a timeslot results in less transmit power available for transmission in each $\mathrm{RB}$, as well as spreads the ACI over multiple RBs.

We introduce the variable $\mathbf{V} \in\{0,1\}^{N \times N \times F \times T}$, where element $V_{i, k, r, t}$ indicates if VUE $i$ and $k$ are scheduled in RBs that are not more than $r$ RBs apart in timeslot $t$. That is, $\forall i, k, r, t$,

$$
V_{i, k, r, t}=\mathbb{1}\left\{X_{i, f, t}=1, X_{k, f^{\prime}, t}=1,\left|f^{\prime}-f\right| \leq r\right\}
$$

Even though we can compute $V_{i, k, r, t}$ from $\mathbf{X}$ directly, in order to preserve linearity, we will instead use the following constraint to enforce (22)

$$
\begin{gathered}
V_{i, k, r, t} \geq X_{i, f, t}+X_{k, f^{\prime}, t}-1 \quad \forall i, k, r, t, f, \\
\max \{f-r, 1\} \leq f^{\prime} \leq \min \{f+r, F\} \\
V_{i, k, r, t} \in\{0,1\} \quad \forall i, k, r, t,
\end{gathered}
$$

Indeed, it is easily checked that if we minimize $V_{i, k, r, t}$ subject to (23), then the solution is given by (22). We will see later that solver of the modified problem strives to minimize $V_{i, k, r, t}$. 


\section{B. Algorithm Description}

The procedure here is an iterative cutting plane method as shown in Algorithm 2. In each iteration we solve [MP] by adding extra cover inequalities as explained below,

1) Modified problem [MP]: The constraint (12b) is the only sensitive constraint in (12). Therefore, we modify the joint scheduling and power control problem formulation in (12) into a scheduling alone problem using the technique explained in Section III variant (ii). We then add constraints (19) and (23) to the problem formulation (12). We call this modified problem as [MP]. During the iterations, we will add robust constraints to the problem as described below.

2) Identifying SINR constraint violations: In each iteration, we solve the problem formulation [MP] and call the resulting solution scheduling matrix as $\mathbf{X}^{[\mathrm{MP}]}$ and successful link status matrix as $\mathbf{Y}^{[\mathrm{MP}]}$. However, not all links that are claimed to be successful in $\mathbf{Y}^{[\mathrm{MP}]}$ are necessarily successful (since [MP] might be not have a complete set of constraints). In fact, the elements of the true successful link status matrix as $\mathbf{Y}^{\text {true }}$ are

$$
Y_{i, j, f, t}^{\mathrm{true}}=\mathbb{1}\left\{\frac{X_{i, f, t}^{[\mathrm{MP}]} \bar{P}_{i, t} H_{i, j}}{\sigma^{2}+\sum_{\substack{k=1 \\ k \neq i}}^{N} \sum_{f^{\prime}=1}^{F} X_{k, f^{\prime}, t^{[\mathrm{MP}]} \lambda_{\left|f^{\prime}-f\right|} \bar{P}_{k, t} H_{k, j}}} \geq \gamma^{\mathrm{T}}\right\} .
$$

A link $(i, j)$ is said to be falsely claimed to be successful in $\mathrm{RB}(f, t)$, if $Y_{i, j, f, t}^{\mathrm{true}}=0$ and $Y_{i, j, f, t}^{[\mathrm{MP}]}=1$.

3) Construction of new constraints: Suppose the link $(\bar{i}, \bar{j})$ scheduled in $\mathrm{RB}(\bar{f}, \bar{t})$ is a falsely claimed to be successful link, i.e., $Y_{\bar{i}, \bar{j}, \bar{f}, \bar{t}}^{\text {true }}=0$ and $Y_{\bar{i}, \bar{j}, \bar{f}, \bar{t}}^{[\mathrm{MP}]}=1$. We will now describe how to construct and add strong cover inequalities to [MP], so that the link $(\bar{i}, \bar{j})$ in $\mathrm{RB}(\bar{f}, \bar{t})$ can not be a falsely claimed to be successful link in any of the future iterations with the current interference scenario. The same procedure for generating cover inequalities will be repeated for all falsely claimed to be successful links.

Let $\mathcal{S}$ be the set of tuples $(k,|f-\bar{f}|)$, where $k \neq \bar{i}$ is an interfering VUE that is scheduled in timeslot $\bar{t}$. Formally,

$$
\mathcal{S}=\left\{(k, r): k \neq \bar{i}, X_{k, f, \bar{t}}^{[\mathrm{MP}]}=1, r=|f-\bar{f}|\right\} .
$$

We see that $\mathcal{S}$ defines an interference scenario for the link $(\bar{i}, \bar{j})$ in $\mathrm{RB}(\bar{f}, \bar{t})$. Since the link $(\bar{i}, \bar{j})$ in $\mathrm{RB}(\bar{f}, \bar{t})$ is a failure, we know that $\sum_{(k, r) \in \mathcal{S}} \lambda_{r} \bar{P}_{k, t} H_{k, j}>\bar{I}_{\bar{i}, \bar{j}}$. We can ensure $Y_{\bar{i}, \bar{j}, \bar{f}, \bar{t}}^{[\mathrm{MP}]}=Y_{\bar{i}, \bar{j}, \bar{f}, \bar{t}}^{\mathrm{true}}$ for the same interference scenario, in all future iterations, by adding the following cover inequality to the problem formulation [MP],

$$
Y_{\bar{i}, \bar{j}, f, \bar{t}} \leq|\mathcal{S}|-\sum_{(k, r) \in \mathcal{S}} V_{\bar{i}, k, r, \bar{t}} \quad \forall f
$$

Observe that, the right hand side of the above cover inequality is zero for the current interference scenario $\mathcal{S}$. Therefore, the above cover inequality enforces $Y_{\bar{i}, \bar{j}, f, \bar{t}}=0, \forall f$, for the same interference scenario in all future iterations.
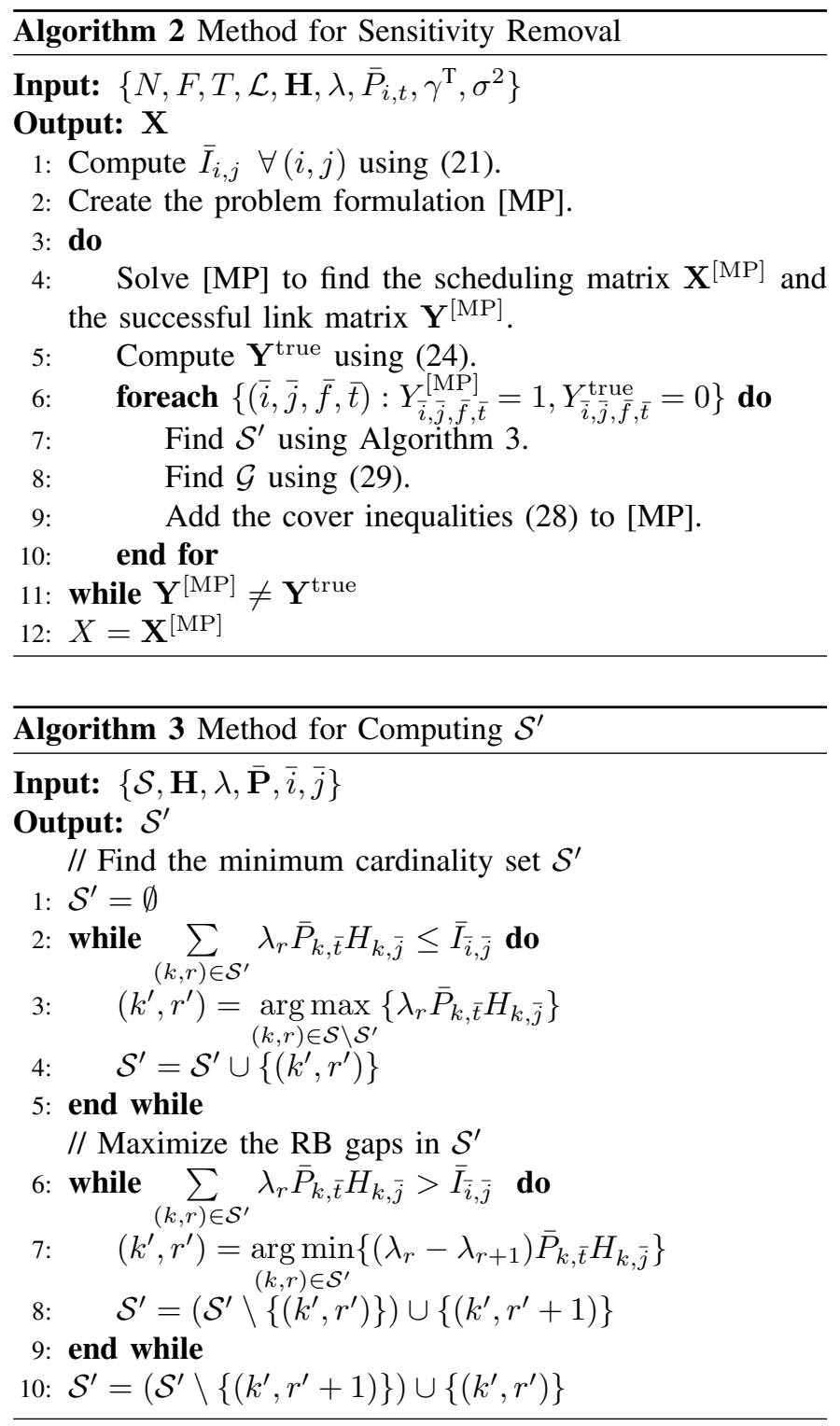

However, we can tighten the cover inequality (26) by replacing $\mathcal{S}$ with $\mathcal{S}^{\prime}$, where $\mathcal{S}^{\prime} \subseteq \mathcal{S}$ is the minimal cardinality set which is sufficient to cause enough interference to make the link $(\bar{i}, \bar{j})$ in $\mathrm{RB}(\bar{f}, \bar{t})$ a failure, i.e., $\sum_{(k, r) \in \mathcal{S}^{\prime}} \lambda_{r} \bar{P}_{k, t} H_{k, j}>$ $\bar{I}_{\bar{i}, \bar{j}}$. In other words, $\mathcal{S}^{\prime}$ is the list of highest interference causing elements within $\mathcal{S}$, i.e.,

$$
\mathcal{S}^{\prime}=\underset{\mathcal{S}^{\prime \prime} \subseteq \mathcal{S}}{\arg \min }\left\{\left|\mathcal{S}^{\prime \prime}\right|: \sum_{(k, r) \in \mathcal{S}^{\prime \prime}} \lambda_{r} \bar{P}_{k, t} H_{k, \bar{j}}>\bar{I}_{\bar{i}, \bar{j}}\right\} .
$$

To tighten the cover inequality further, we increment the RB gaps in $\mathcal{S}^{\prime}$ to maximally possible values, in such a way that any further increment of any RB gaps in the resulting $\mathcal{S}^{\prime}$ would result in insufficient interference to break the link $(\bar{i}, \bar{j})$ in $\mathrm{RB}$ $(\bar{f}, \bar{t})$. The computation of $\mathcal{S}^{\prime}$ is explained in Algorithm 3 .

The final expression for the constraints to be added to [MP] due to the falsely claimed to be successful link $(\bar{i}, \bar{j})$ in RB 


$$
\begin{aligned}
& (\bar{f}, \bar{t}) \text { is } \\
& (|\mathcal{G}|+1) Y_{\bar{i}, \bar{j}, f, \bar{t}} \leq\left|\mathcal{S}^{\prime}\right|+|\mathcal{G}|-\sum_{(k, r) \in \mathcal{S}^{\prime} \cup \mathcal{G}} V_{\bar{i}, k, r, \bar{t}} \quad \forall f
\end{aligned}
$$

where the set $\mathcal{G}$ is for lifting the cover inequality (28) further [49]. Here, $\mathcal{G}$ is the set $\left(k^{\prime}, r^{\prime}\right)$ tuples, where VUE $k^{\prime}$ would cause more interference to the link $(\bar{i}, \bar{j})$ than any interferer in $\mathcal{S}$. Formally, for $k \in \mathcal{N}$ and $r^{\prime} \in\{0,1, \ldots, F-1\}$,

$$
\mathcal{G}=\left\{\left(k^{\prime}, r^{\prime}\right): \lambda_{r^{\prime}} \bar{P}_{k^{\prime}, t} H_{k^{\prime}, \bar{j}}>\max _{(k, r) \in \mathcal{S}}\left\{\lambda_{r} \bar{P}_{k, t} H_{k, \bar{j}}\right\}\right\} .
$$

We note that VUE $k^{\prime}$ is not necessarily scheduled according to $\mathrm{X}^{[\mathrm{MP}]}$. Furthermore, we note from (28) that increasing the value of $V_{\bar{i}, k, r, \bar{t}}$ decreases the feasible region (hence, does not improve the objective value). Therefore, the solver always strive to minimize the value of $V_{\bar{i}, k, r, \bar{t}}$, which implicitly enforces the relation between $V_{\bar{i}, k, r, \bar{t}}$ and $\mathbf{X}$ as explained in Sec. V-A. Finally, we observe that the cover inequality (28) can be applied for all timeslots $t$, if $\bar{P}_{i, t}=\bar{P}_{i, t^{\prime}}, \forall i, t, t^{\prime}$.

\section{Vi. Performance Evaluation}

\section{A. Scenario and Parameters}

The simulation parameters are summarized in Table II. For simulation purposes and ease of reproducibility, we evaluate the proposed algorithms in a fairly simplistic network topology. Of course, the proposed algorithms do not depend on any particular network topology or simulation parameter values.

The network topology consists of $N$ VUEs in a convoy. For ease of simulation and reproducibility, we consider a statistical model for VUEs' topology. Researchers conclude that the vehicular topologies are different in different scenarios [50]-[54]. E.g., in a dense traffic scenario, VUEs usually keep a fixed distance with the adjacent VUEs, however, for sparse traffic, VUEs usually follow a modified Poisson point process on a lane, i.e., the distance between any two adjacent VUEs follows a shifted exponential distribution with minimum distance $d_{\min }$ and average distance $d_{\text {avg }}$ [50]. For simulation purposes, we consider a freeway/highway scenario, hence the adjacent vehicular distances $d$ is modelled as a random variable with probability density function,

$$
f(d)= \begin{cases}\left(d_{\text {avg }}-d_{\min }\right)^{-1} \exp \left(-\frac{d-d_{\min }}{d_{\text {avg }}-d_{\min }}\right), & d \geq d_{\min } \\ 0, & \text { otherwise }\end{cases}
$$

We choose $d_{\text {avg }}=48.6 \mathrm{~m}$, which corresponds to $2.5 \mathrm{~s}$ for a vehicular speed of $70 \mathrm{~km} / \mathrm{h}$, as recommended by $3 \mathrm{GPP}[55$, section A.1.2] for freeway scenario, and let $d_{\min }=10 \mathrm{~m}$. We note that the exact vehicular mobility model is less of a concern for the time scale of the problem under study. Typically, the scheduling time horizon (which is related to the latency requirement) is less than $100 \mathrm{~ms}$, over which time the slow channel state information (i.e., pathloss and shadowing) does not vary significantly, even at highway speeds. Hence, there is no strong need to model mobility during the scheduling interval ( $T$ timeslots).

We adopted the channel model from [56], which is a model based on the real-time measurements of $\mathrm{V} 2 \mathrm{~V}$ links at carrier
TABLE II: System Simulation Parameters

\begin{tabular}{ll}
\hline Parameter & Value \\
\hline ACIR model & $3 \mathrm{GPP}$ mask [60] \\
$\gamma^{\mathrm{T}}$ & $5 \mathrm{~dB}$ \\
$P^{\max }$ & $24 \mathrm{dBm}$ \\
$\mathrm{PL}_{0}$ & $63.3 \mathrm{~dB}$ \\
$n$ & 1.77 \\
$d_{0}$ & $10 \mathrm{~m}$ \\
$\sigma_{1}$ & $3.1 \mathrm{~dB}$ \\
Penetration Loss & $10 \mathrm{~dB}$ per obstructing VUE \\
$\sigma^{2}$ & $-95.2 \mathrm{dBm}$ \\
$d_{\text {avg }}$ & $48.6 \mathrm{~m}$ \\
$d_{\text {min }}$ & $10 \mathrm{~m}$ \\
$\eta$ & $\gamma^{\mathrm{T}}\left(N P^{\max }+\sigma^{2}\right)$ \\
\hline
\end{tabular}

frequency $5.2 \mathrm{GHz}$ in a highway scenario, and in line with the measurements done in [57]-[59]. The pathloss model in [56] is

$$
\mathrm{PL}(d)=\mathrm{PL}_{0}+10 n \log _{10}\left(d / d_{0}\right)+X_{\sigma_{1}}
$$

where $d$ is the distance, $n$ is the pathloss exponent, $\mathrm{PL}_{0}$ is the pathloss at a reference distance $d_{0}$, and $X_{\sigma_{1}}$ represents the shadowing effect modeled as a zero-mean Gaussian random variable with standard deviation $\sigma_{1}$. Presently, there is a lack of enough measurements for the penetration loss caused by multiple obstructing vehicles. However, the penetration loss caused by single vehicle has been widely studied. Measurements show that an obstructing truck causes $12-13 \mathrm{~dB}$ [9], a bus $15-20 \mathrm{~dB}$ [11], a van $20 \mathrm{~dB}$ [10], and a car $10 \mathrm{~dB}$ [8] penetration loss. For the simulation purpose, we assume penetration loss of $10 \mathrm{~dB}$ for each obstructing VUE.

For concreteness, we adopt the LTE specification for the RB size, noise variance, maximum transmit power and ACIR [60]. Hence, $\sigma^{2}=-95.2 \mathrm{dBm}$ and $P^{\max }=24 \mathrm{dBm}$. Moreover, the ACIR values $\lambda_{r}$ is chosen the same as the mask specified by 3GPP [60],

$$
\lambda_{r}= \begin{cases}1, & r=0 \\ 10^{-3}, & 1 \leq r \leq 4 . \\ 10^{-4.5}, & \text { otherwise }\end{cases}
$$

We assume that $\gamma^{\mathrm{T}}=5 \mathrm{~dB}$ is sufficient for a transmission to be successful (i.e., that the packet error probability averaged over the small-scale fading is sufficiently small to satisfy the reliability constraint). We note that this corresponds to a fairly small number of information bits (i.e., small message size). Longer messages, e.g., CAM messages, requires increased transmit power, which might not be possible due to regulatory constraints, or a larger RB size. Increasing the RB size in frequency will reduce the problem with $\mathrm{ACI}$, since there is less need to multiplex transmissions in frequency. However, since the noise variance is proportional to the RB bandwidth, this will also cause the SNR and the maximum transmission range to be reduced. Increasing the RB size in time will not affect the SNR, but will reduce the number of available timeslots $T$ (for a fixed latency requirement). 
As the number of VUEs in the network increases, we need to reuse timeslots. To avoid excessive CCI, we can (i) form groups (cells) of $N$ adjacent VUEs, (ii) form clusters of adjacent groups, (iii) divide the available timeslots among the groups in each cluster, and (iv) schedule the VUEs in each group independently. In this arrangement, CCI can occur between clusters but not between the groups in a cluster. The intercluster interference can be made negligible by making the distance between groups that use the same timeslots, i.e, the reuse distance, large enough. However, if there are $K$ groups in a cluster, the number of timeslots available for the scheduler in a group is $T=T_{\text {tot }} / K$, where $T_{\text {tot }}$ is the total number of available timeslots, which in turn is equal to the latency constraint divided by the RB time duration. Moreover, the number of VUEs that the scheduler needs to consider can be chosen to a fixed number $N$, even as the number of VUEs in the network becomes arbitrarily large.

From the discussion above, we conclude that the value of $T$ becomes smaller when (i) latency requirement becomes small, (ii) timeslots needs to be reused, or (iii) the message size increases. The value of $T$ can therefore be fairly small in practical situations. With this in mind, and since we are interested in evaluating the impact of ACI in limiting scenarios, we will study performance for small values of $T$. It should be clear that the simulations below are relevant also for the timeslot reuse scheme above (for sufficiently long reuse distances). Moreover, the simulations are also relevant when we enable transmission of longer messages by increasing the RB size in time: this would not change the maximum transmission range (for fixed $P^{\max }, \sigma^{2}$, and $\gamma^{\mathrm{T}}$ ) or the interference generated by a transmission (which depends on the transmit power and $\lambda_{r}$ ).

For simulation purposes, we choose the set of intended receivers $\mathcal{R}_{i}$ for a transmitting VUE $i$ as all the other $N-1$ VUEs in the network. The value of $C$ in column generation method in Section IV is set to 10 , which turns out to be sufficiently large such that the while loop in Alg. 1 terminates before reaching the maximum number of iterations in our simulations.

\section{B. Simulation Results}

We compare algorithms using the following performance metrics

$$
\begin{aligned}
Z_{i} & =\sum_{j \in \mathcal{R}_{i}} Z_{i, j}, \\
\bar{Z}_{i} & =\mathrm{E}\left[Z_{i}\right], \\
\bar{Z} & =\frac{1}{N} \sum_{i=1}^{N} \bar{Z}_{i},
\end{aligned}
$$

where $Z_{i}$ is the number of successful links from VUE $i$ to VUEs in $\mathcal{R}_{i}$. The quantity $\bar{Z}_{i}$ is the expected value of $Z_{i}$, where the expectation is taken over the random quantities in the experiment, i.e., the inter-VUE distances and shadow fading. Finally, $\bar{Z}$ is the number of successful links for a VUE, averaged across all VUEs. In other words, the metric $\bar{Z}$ can be interpreted as the average number of receiving VUEs that can decode a packet from a certain VUE. Clearly, $Z_{i}$ must be sufficiently large to support the application in mind. However, to specify requirements on $Z_{i}$ is out of scope of this paper.

For scheduling-only algorithms, we use equal transmit power for all VUEs. In fact, we let $\bar{P}_{i, t}=P^{\max } \forall i, t$, since equal-power scheduling performance increases with transmit power, as proved in [41]. Moreover, we used the Gurobi toolbox [62] along with Matlab for solving all MBLP and BLP problems.

The compared algorithms are summarized in Table III. As a baseline method, we show the results of the ACI-aware heuristic scheduling algorithm proposed in our previous work [41], and ACI-unaware multicast scheduling algorithm from [61]. To the best of out knowledge, there is no study upon multicast scheduling for maximizing the connectivity in the existing literature, other than [41]. The proposed multicast scheduling in [61] is for maximizing the QoS, hence, we modify its objective to maximize the connectivity, and present the result here as black colored line marked with plus. We further note that the proposed heuristic scheduling algorithms in [41] and [61] have polynomial computational complexity, whereas the scheduling problem (12) variant (ii) (i.e., optimal scheduling (numerical)) has exponential computational complexity.

Due to the numerical sensitivity of the problem (12) variant (ii), a numerical solution may not provide optimal performance. Therefore, we refer the solutions provided by the solver as optimal scheduling (numerical), and present the results as the green-colored curves marked with triangles in Figs. 3-4. In order to quantify the optimality gap, we have computed optimal solution for the scheduling problem by using the sensitivity reduction techniques explained in Section V, and the results are shown in blue-colored curves marked with circles. Observe that the gap between green and blue curves in Figs. 3-4 indicate the optimality gap, i.e., performance degradation due to the sensitivity of the problem. The magenta-colored curves marked with diamonds indicate the performance of joint scheduling and power control using the column generation method as explained in Section IV. The red curves marked with stars are the performance for joint scheduling and power control upon solving (12) in its exact form using the Gurobi toolbox [62]. However, due to the sensitivity of the problem formulation, the Gurobi solver may provide near-optimal solutions instead of optimal solutions. As seen from Figs. 3-4, our column generation method performs close to the joint scheduling and power control (numerical) solution, with less computational complexity.

In Fig. 3(a), we plot $\bar{Z}$ versus number of timeslots $T$. We observe that $\bar{Z}$ saturates to around 6 successful links per VUE as $T$ increases. This is because links beyond the $3^{\text {rd }}$ neighbor on each side of a transmitting VUE tend to be noise limited, chiefly due to the high penetration loss of the intermediate 


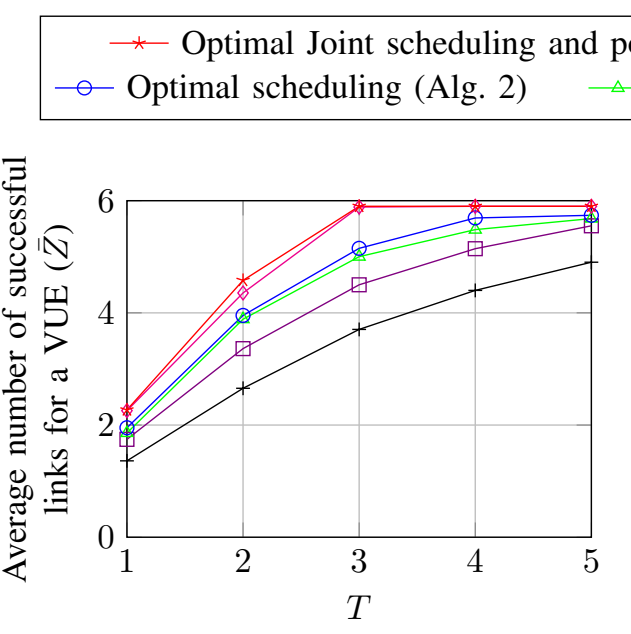

(a) $(F=20, N=20)$

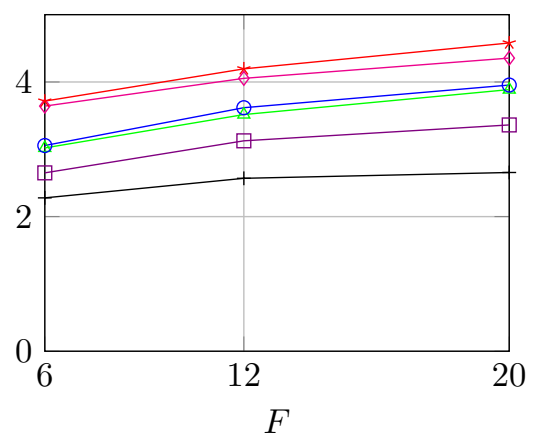

(b) $(T=2, N=20)$

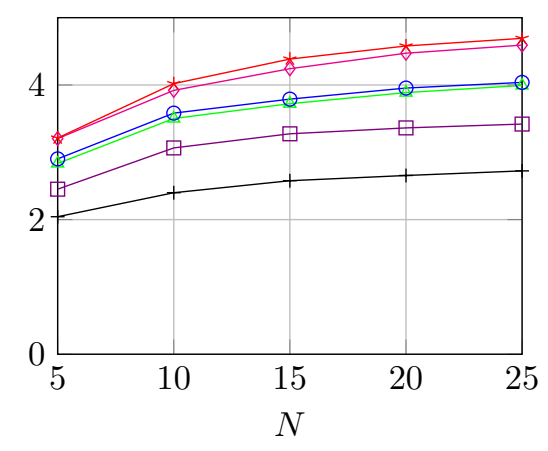

(c) $(F=20, T=2)$

Fig. 3: Average number of successful links for a VUE $(\bar{Z})$ for various algorithms

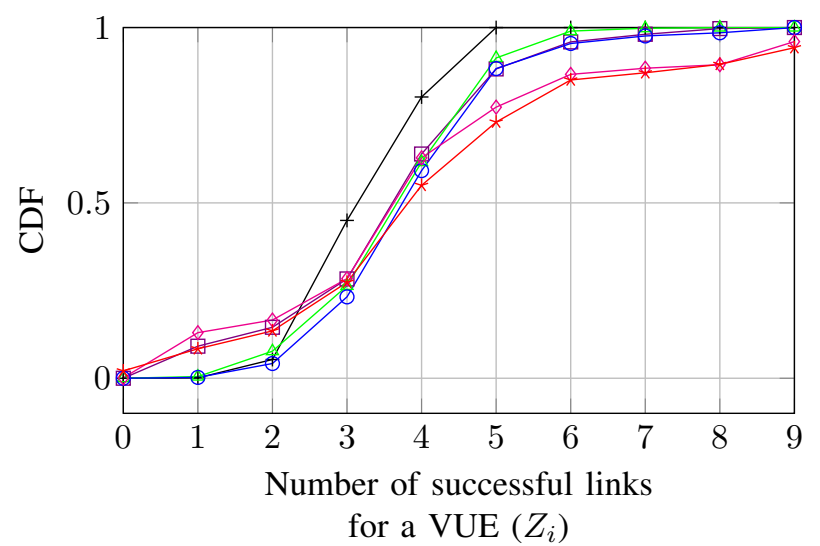

(a)

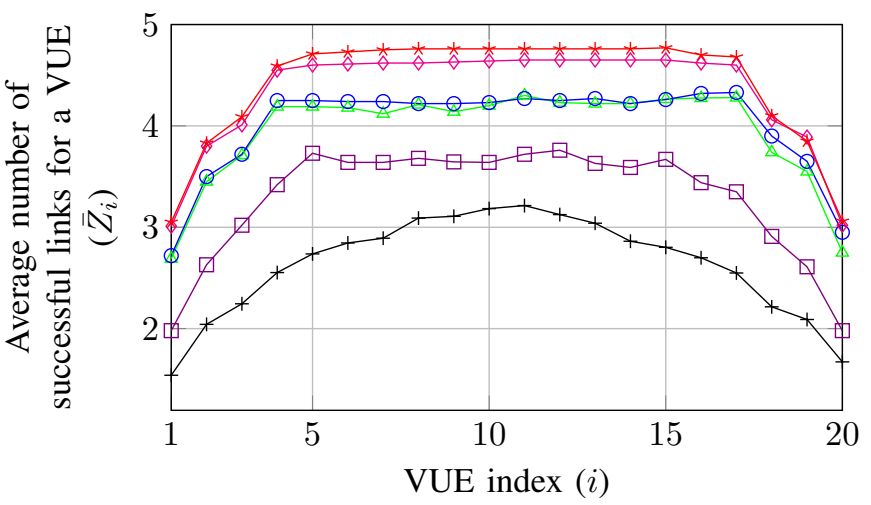

(b)

Fig. 4: Fairness comparison for number of successful links $(F=20, T=2, N=20)$

VUEs ${ }^{4}$. This also implies that if ACI can be completely avoided, each VUE can communicate to approximately 6 neighboring VUEs when there are sufficient number of RBs (i.e., $F T \geq N$ ).

One way to reduce the impact of ACI is to increase the number of frequency slots $F$, thus allowing larger spacing between VUEs in frequency. However, as shown in Fig. 3(b), the performance increases only marginally as we increase $F$. A closer inspection of the simulation results for the case when $F=6$ or $F=12$ reveals that multiple VUEs are scheduled in the same RB, thereby causing CCI. In Fig. 3(c), we show the performance for various number of VUEs $N$. As we increase $N$, the performance improves since more and more receivers are becoming available for each transmission. The value of $T$ is chosen to be 2 for Figs. 3(b)-(c) and Fig. 4,

${ }^{4}$ For example, VUE $i$ is connected to VUE $j$ if and only if the channel gain $H_{i, j} \geq \gamma^{\mathrm{T}}+\sigma^{2}-P^{\max }=-114.2 \mathrm{~dB}$. However, if VUE $i$ and $j$ are blocked by 3 intermediate VUEs, then the penetration loss of $30 \mathrm{~dB}$ makes the channel gain value less than $-114.2 \mathrm{~dB}$ when the shadowing gain is not above $0 \mathrm{~dB}$ and adjacent VUEs are at distance $d_{\text {avg }}$ from each other. since $T=1$ would make scheduling only half of the VUEs in half-duplex scenario and higher values of $T$ reduces the ACI problem, eventually to the point when all algorithms performs equally for low number of VUEs, i.e., $N \leq 20$ (see Fig. 3(a)). Observe that the joint scheduling and power control provides noticeable improvement in performance compared to the scheduling-alone algorithms, as seen from Figs. 3-4.

To compare the fairness of the schemes, we plot the CDF for $\left\{Z_{i}\right\}_{i=1}^{N}$ and $\bar{Z}_{i}=\mathrm{E}\left[Z_{i}\right]$ in Fig. 4(a) and Fig. 4(b), respectively. We can conclude that performance is approximately the same for the VUEs in the center of the network, but falls off for the VUEs on either edge of the network. This is to be expected, since links become noise-limited as the number of blocking VUEs increases and VUEs at the edge of the network have fewer close-by neighbors. Hence, it seems that we do not need to explicitly enforce fairness, even though this can be done as explained in (12) variant (iv).

We note that, when $N \leq F T$, an ACI-unaware scheduling and power control scheme is trivial in a full duplex scenario, 
TABLE III: Summary of compared algorithms

\begin{tabular}{|c|c|c|c|c|}
\hline Line Style & Scheduling & Power & Complexity (worst-case) & Algorithm \\
\hline * & Optimized & Optimized & $\mathcal{O}\left(\frac{\left(N^{2}+N F T\right)^{3} 2^{(N+1) N F T}}{\log \left(N^{2}+N F T\right)}\right)$ & (12) \\
\hline$\diamond$ & Optimized & Optimized & $\mathcal{O}\left(\frac{T\left(N^{2}+N F\right)^{3} 2^{(N+1) N F}}{\log \left(N^{2}+N F\right)}\right)$ & Alg. 1 \\
\hline 0 & Optimized & Equal & $\mathcal{O}\left(2^{(2 N+1) N F T)}\right)$ & Alg. 2 \\
\hline$\triangle$ & Optimized & Equal & $\mathcal{O}\left(2^{(N+1) N F T)}\right)$ & (12) variant (ii) \\
\hline$\square$ & Optimized & Equal & $\mathcal{O}\left(N F T\left(F T+N^{2}\right)\right)$ & {$[41]$} \\
\hline 1 & Optimized & Equal & $\mathcal{O}\left(N^{2} F T\right)$ & [61] \\
\hline
\end{tabular}

i.e., schedule all VUEs in non-overlapping RBs, and allocate maximum transmit power to each VUE. For half duplex case, the performance also depends upon the way we assign transmitter/receiver status to each VUE. For instance, if VUE is scheduled in timeslot $t$, then we should avoid scheduling any VUEs in $\mathcal{R}_{i}$. If this is possible, then the schedule is optimal. However, ignoring ACI would lead to significant performance reduction as seen from the results of scheduling algorithm in [61] in Figs. 3-4.

\section{CONCLUSIONS}

This paper studies the performance of direct V2V broadcast communication in the presence of CCI and ACI. The main focus is on scenarios where $\mathrm{ACI}$ is comparable or dominant over $\mathrm{CCI}$, e.g., when there are enough RBs to schedule VUEs with relative large RB-reuse distance. From the results presented in this paper, which are for half-duplex communication, we can draw the following conclusions,

1) Performance is mainly limited by ACI when VUEs are multiplexed in frequency. However, proper scheduling and power control schemes can be used to mitigate the impact of ACI.

2) The joint scheduling and power control problem to maximize the VUE connectivity, in the presence of ACI, can be formulated as a mixed Boolean linear programming (MBLP) problem. From this problem formulation, we can derive a scheduling-alone algorithm as a boolean linear programming (BLP) problem and a power-control-alone algorithm as a MBLP problem.

3) The computational complexity of the joint scheduling and power control problem can be reduced using a column generation approximation method with a minor compromise on performance.

4) Due to the high dynamic range of channel and ACI values, the scheduling problem is numerically sensitive, and the optimization solver will typically return nearoptimal solutions instead of optimal solutions. However, the sensitivity can be removed by applying cover inequalities to the original problem formulation. Very close to optimal scheduling can thereby be computed at the price of increased computational complexity.

5) Optimal joint scheduling and power control performs noticeably better than optimal, equal-power scheduling.
6) Fairness can be explicitly enforced, but this seems unnecessary as the proposed algorithms provide good fairness anyways.

Finally, we note that the high computational complexity for finding the optimal solution might limit the practical utility of the proposed algorithms for larger networks. However, the results of the optimal scheme can be used by future researchers to benchmark and develop more robust algorithms with low computational complexity. Our future work focus upon developing low-complexity algorithms, addressing the scalability issues, and developing a distributed algorithm to remove the need for a centralized controller.

\section{REFERENCES}

[1] 3GPP, "Intelligent Transport Systems (ITS); Vehicular Communications; Basic Set of Applications; Part 2: Specification of Cooperative Awareness Basic Service,” TR 102.637, Mar. 2011. [Online]. Available: https://www.etsi.org/deliver/etsi_ts/102600_102699/10263701/

[2] _ - "Intelligent Transport Systems (ITS); Vehicular Communications; Basic Set of Applications; Part 3: Specifications of Decentralized Environmental Notification Basic Service," 3rd Generation Partnership Project (3GPP), TR 302.637, Apr. 2019. [Online]. Available: https://www.etsi.org/deliver/etsi_en/302600_302699/30263702/

[3] W. Sun, E. G. Ström, F. Brännström, K. C. Sou, and Y. Sui, "Radio resource management for D2D-based V2V communication," IEEE Transactions on Vehicular Technology, vol. 65, no. 8, pp. 6636-6650, Aug 2016.

[4] A. Mohammadi and F. M. Ghannouchi, Transmitter Design for MIMO Wireless Communications. Springer Berlin Heidelberg, 2012, pp. 129149.

[5] P. M. Lavrador, T. R. Cunha, P. M. Cabral, and J. C. Pedro, "The linearity-efficiency compromise," IEEE Microwave Magazine, vol. 11, no. 5, pp. 44-58, Aug 2010.

[6] P. B. Kenington, "Linearized transmitters: an enabling technology for software defined radio," IEEE Communications Magazine, vol. 40, no. 2, pp. 156-162, Feb 2002.

[7] J. Chani-Cahuana, P. N. Landin, C. Fager, and T. Eriksson, "Iterative learning control for RF power amplifier linearization," IEEE Transactions on Microwave Theory and Techniques, vol. 64, no. 9, pp. 27782789, Sept 2016.

[8] T. Abbas, F. Tufvesson, K. Sjöberg, and J. Karedal, "A measurement based shadow fading model for vehicle-to-vehicle network simulations," International Journal of Antennas and Propagation, 052015.

[9] D. Vlastaras, T. Abbas, M. Nilsson, R. Whiton, M. Olbäck, and F. Tufvesson, "Impact of a truck as an obstacle on vehicle-to-vehicle communications in rural and highway scenarios," in Proc. IEEE 6th International Symposium on Wireless Vehicular Communications, Vancouver, Canada, 2014.

[10] R. Meireles, M. Boban, P. Steenkiste, O. Tonguz, and J. Barros, "Experimental study on the impact of vehicular obstructions in VANETs," in 2010 IEEE Vehicular Networking Conference, Dec 2010, pp. 338-345.

[11] R. He, A. F. Molisch, F. Tufvesson, Z. Zhong, B. Ai, and T. Zhang, "Vehicle-to-vehicle propagation models with large vehicle obstructions," IEEE Transactions on Intelligent Transportation Systems, vol. 15, no. 5, pp. 2237-2248, Oct 2014 
[12] C. H. Yu, K. Doppler, C. B. Ribeiro, and O. Tirkkonen, "Resource sharing optimization for device-to-device communication underlaying cellular networks," IEEE Trans. Wireless Commun., vol. 10, pp. 27522763, 2011.

[13] M. I. Ashraf, C. Liu, M. Bennis, W. Saad, and C. S. Hong, "Dynamic resource allocation for optimized latency and reliability in vehicular networks," IEEE Access, vol. 6, pp. 63 843-63 858, 2018.

[14] R. Molina-Masegosa, J. Gozalvez, and M. Sepulcre, "An IEEE 802.11passisted LTE-V scheduling for reliable multi-link V2X communications," in 2018 IEEE 88th Vehicular Technology Conference (VTC-Fall), Aug 2018, pp. 1-5.

[15] J. Lv, X. He, J. Li, H. Wang, and T. Luo, "Transmission capacity analysis of distributed scheduling in lte-v2v mode 4 communication," in Communications and Networking, X. Liu, D. Cheng, and L. Jinfeng, Eds. Cham: Springer International Publishing, 2019, pp. 225-234.

[16] T. Sahin and M. Boban, "Radio resource allocation for reliable out-ofcoverage v2v communications," in 2018 IEEE 87th Vehicular Technology Conference (VTC Spring), June 2018, pp. 1-5.

[17] J. Zhou, R. Q. Hu, and Y. Qian, "Message scheduling and delivery with vehicular communication network infrastructure," in 2013 IEEE Global Communications Conference (GLOBECOM), Atlanta, USA, Dec 2013, pp. 575-580

[18] J. He, Z. Tang, Z. Fan, and J. Zhang, "Enhanced collision avoidance for distributed lte vehicle to vehicle broadcast communications," IEEE Communications Letters, vol. 22, no. 3, pp. 630-633, March 2018.

[19] X. Zhang, Y. Shang, X. Li, and J. Fang, "Research on overlay d2d resource scheduling algorithms for v2v broadcast service," in 2016 IEEE 84th Vehicular Technology Conference (VTC-Fall), Sep. 2016, pp. 1-5.

[20] R. Fritzsche and A. Festag, "Location-based scheduling for cellular v2v systems in highway scenarios," in 2018 IEEE 87th Vehicular Technology Conference (VTC Spring), June 2018, pp. 1-5.

[21] C. Zhang, Y. Zang, J. A. L. Calvo, and R. Mathar, "A novel v2v assisted platooning system: Control scheme and mac layer designs," in 2017 IEEE 28th Annual International Symposium on Personal, Indoor, and Mobile Radio Communications (PIMRC), Oct 2017, pp. 1-7.

[22] F. Zeng, R. Zhang, X. Cheng, and L. Yang, "Channel prediction based scheduling for data dissemination in VANETs," IEEE Communications Letters, vol. 21, no. 6, pp. 1409-1412, 2017.

[23] V. K. Shrivastava, P. Makhija, and R. Raj, "Joint optimization of energy efficiency and scheduling strategies for side-link relay system," in 2017 IEEE Wireless Communications and Networking Conference (WCNC), March 2017, pp. 1-6.

[24] J. Tian and C. Lv, "Connectivity based transmit power control in vanet," in 2012 8th International Wireless Communications and Mobile Computing Conference (IWCMC), Aug 2012, pp. 505-509.

[25] H. Zhang, Y. Ma, D. Yuan, and H. Chen, "Quality-of-service driven power and sub-carrier allocation policy for vehicular communication networks," IEEE Journal on Selected Areas in Communications, vol. 29, no. 1, pp. 197-206, January 2011

[26] Q. Wang, P. Fan, and K. B. Letaief, "On the joint v2i and v2v scheduling for cooperative vanets with network coding," IEEE Transactions on Vehicular Technology, vol. 61, no. 1, pp. 62-73, Jan 2012.

[27] M. Jonsson, K. Kunert, and A. Böhm, "Increasing the probability of timely and correct message delivery in road side unit based vehicular communication," in 2012 15th International IEEE Conference on Intelligent Transportation Systems, Sep. 2012, pp. 672-679.

[28] J. Wang, Y. Ji, X. Wang, and F. Liu, "Rsu-coordinated multi-channel mac with multi-criteria channel allocation," in 2012 International Conference on Connected Vehicles and Expo (ICCVE), Dec 2012, pp. 60-65.

[29] A. Hisham, W. Sun, E. G. Ström, and F. Brännström, "Power control for broadcast $\mathrm{V} 2 \mathrm{~V}$ communications with adjacent carrier interference effects," in IEEE International Conference on Communications (ICC), May 2016.

[30] H. Albasry, H. Zhu, and J. Wang, "The impact of in-band emission interference in D2D-enabled cellular networks," in GLOBECOM 2017 2017 IEEE Global Communications Conference, Dec 2017.

[31] W. Li, J. Chen, H. Long, and B. Wu, "Performance and analysis on LTE system under adjacent channel interference of broadcasting system," in 2012 IEEE 12th International Conference on Computer and Information Technology, Chengdu, China, Oct 2012, pp. 290-294.

[32] Q. Wang and X. Li, "Analysis of LTE FDD and TD-LTE combination network's interference," in 2016 2nd IEEE International Conference on Computer and Communications (ICCC), Chengdu, China, Oct 2016, pp. 2332-2336.
[33] J. Ribadeneira-Ramirez, G. Martinez, D. Gomez-Barquero, and N. Cardona, "Interference analysis between digital terrestrial television (DTT) and 4G LTE mobile networks in the digital dividend bands," IEEE Transactions on Broadcasting, vol. 62, no. 1, pp. 24-34, March 2016.

[34] Electronic Communications Committee (ECC), "CEPT Report 40: Compatibility study for LTE and WiMAX operating within the bands $880-915 \mathrm{MHz} / 925-960 \mathrm{MHz}$ and $1710-1785 \mathrm{MHz} / 1805-1880 \mathrm{MHz}$ (900/1800 MHz bands)," Tech. Rep., Nov 2010. [Online]. Available: https://www.ecodocdb.dk/download/4ce187bc-3569/CEPTREP040.PDF

[35] K. Xia, Y. Wang, and D. Zhang, "Coexistence interference evaluation and analysis of LTE with 3D-MIMO system," in 2017 IEEE 28th Annual International Symposium on Personal, Indoor, and Mobile Radio Communications (PIMRC), Oct 2017, pp. 1-6.

[36] L. Wang, X. Qi, and K. Wu, "Embracing adjacent channel interference in next generation WiFi networks," in 2016 IEEE International Conference on Communications (ICC), Kuala Lumpur, Malaysia, May 2016.

[37] A Adya, P. Bahl, J. Padhye, A. Wolman, and L. Zhou, "A multiradio unification protocol for IEEE 802.11 wireless networks," in First International Conference on Broadband Networks, San Jose, CA, USA, Oct 2004, pp. 344-354.

[38] J. Nachtigall, A. Zubow, and J. P. Redlich, "The impact of adjacent channel interference in multi-radio systems using IEEE 802.11," in 2008 International Wireless Communications and Mobile Computing Conference, Crete Island, Greece, Aug 2008, pp. 874-881.

[39] C. Campolo, A. Molinaro, and A. Vinel, "Understanding adjacent channel interference in multi-channel VANETs," in 2014 IEEE Vehicular Networking Conference (VNC), Dec 2014, pp. 101-104.

[40] C. Campolo, C. Sommer, F. Dressler, and A. Molinaro, "On the impact of adjacent channel interference in multi-channel VANETs," in 2016 IEEE International Conference on Communications (ICC), May 2016.

[41] A. Hisham, E. G. Ström, F. Brännström, and L. Yan, "Scheduling and power control for $\mathrm{V} 2 \mathrm{~V}$ broadcast communications with co-channel and adjacent channel interference," IEEE Access, vol. 7, pp. 67 041-67 058, Jun. 2019.

[42] 3GPP, "Evolved Universal Terrestrial Radio Access (E-UTRA); Physical channels and modulation," 3rd Generation Partnership Project (3GPP), TR 36.211, March 2017. [Online]. Available: http://www.3gpp.org/ftp/Specs/archive/36_series/36.211

[43] W. Xu, H. Zhou, N. Cheng, F. Lyu, W. Shi, J. Chen, and X. Shen, "Internet of vehicles in big data era," IEEE/CAA Journal of Automatica Sinica, vol. 5, no. 1, pp. 19-35, Jan 2018.

[44] F. Yang, S. Wang, J. Li, Z. Liu, and Q. Sun, "An overview of internet of vehicles," China Communications, vol. 11, no. 10, pp. 1-15, Oct 2014

[45] P. Björklund, P. Värbrand, and D. Yuan, "A column generation method for spatial TDMA scheduling in ad hoc networks," Ad Hoc Networks, vol. 2, pp. 405-418, 2004.

[46] A. Florian, Potra, and J. W. Stephen, "Interior-point methods," Journal of Computational and Applied Mathematics, vol. 124, no. 1, pp. 281302, Dec. 2000

[47] V. V. Vazirani, Approximation Algorithms. Berlin, Heidelberg: SpringerVerlag, 2001

[48] A. Capone, L. Chen, S. Gualandi, and D. Yuan, "A new computationa approach for maximum link activation in wireless networks under the SINR model," IEEE Transactions on Wireless Communications, vol. 10 no. 5, pp. 1368-1372, May 2011.

[49] Z. Gu, G. L. Nemhauser, and M. W. Savelsbergh, "Lifted flow cover inequalities for mixed 0-1 integer programs," Mathematical Programming, vol. 85, no. 3, pp. 439-467, Aug 1999. [Online]. Available: https://doi.org/10.1007/s101070050067

[50] R. T. Luttinen, "Statistical analysis of vehicle time headways," Ph.D. dissertation, 1996. [Online]. Available: http://urn.fi/urn:nbn:fi:tkk007970

51] V. Naumov, R. Baumann, and T. Gross, "An evaluation of inter-vehicle ad hoc networks based on realistic vehicular traces," in Proceedings ACM International Aymposium on Mobile Ad Hoc Networking and Computing. ACM Press, 2006, pp. 108-119.

[52] S. Uppoor and M. Fiore, "Large-scale urban vehicular mobility for networking research," in 2011 IEEE Vehicular Networking Conference $(V N C)$, Nov 2011, pp. 62-69.

[53] H. Huang, P. Luo, M. Li, D. Li, X. Li, W. Shu, and M. Wu, "Performance evaluation of SUVnet with real-time traffic data," IEEE Transactions on Vehicular Technology, vol. 56, no. 6, pp. 3381-3396, Nov 2007.

[54] D. Kong and X. Guo, "Analysis of vehicle headway distribution on multi-lane freeway considering car-truck interaction," Advances in 
Mechanical Engineering, vol. 8, no. 4, 2016. [Online]. Available: https://doi.org/10.1177/1687814016646673

[55] 3GPP, "Technical specification group radio access network; study on LTE-based V2X services," 3rd Generation Partnership Project (3GPP), TR 36.885, June 2016. [Online]. Available: http://www.3gpp.org/ftp/Specs/html-info/36885.htm

[56] J. Karedal, N. Czink, A. Paier, F. Tufvesson, and A. Molisch, "Path loss modeling for vehicle-to-vehicle communications," IEEE Transactions on Vehicular Technology, vol. 60, no. 1, pp. 323-328, Jan. 2011.

[57] T. Abbas, J. Nuckelt, T. Kürner, T. Zemen, C. F. Mecklenbräuker, and F. Tufvesson, "Simulation and measurement-based vehicle-to-vehicle channel characterization: Accuracy and constraint analysis," IEEE Transactions on Antennas and Propagation, vol. 63, no. 7, pp. 3208-3218, July 2015.

[58] L. Cheng, B. E. Henty, F. Bai, and D. D. Stancil, "Highway and rura propagation channel modeling for vehicle-to-vehicle communications at $5.9 \mathrm{GHz}$," in 2008 IEEE Antennas and Propagation Society International Symposium, July 2008

[59] J. Kunisch and J. Pamp, "Wideband car-to-car radio channel measurements and model at $5.9 \mathrm{GHz}$," in Proceedings IEEE Vehicular Technology Conference, Sept 2008

[60] 3GPP, "Evolved Universal Terrestrial Radio Access (E-UTRA); Radio Frequency (RF) system scenarios,” TR 36.942, Oct. 2014. [Online]. Available: http://www.3gpp.org/ftp/Specs/html-info/36942.htm

[61] B. Peng, C. Hu, T. Peng, Y. Yang, and W. Wang, "A resource allocation scheme for D2D multicast with QoS protection in OFDMA-based systems," in 2013 IEEE 24th Annual International Symposium on Personal, Indoor, and Mobile Radio Communications (PIMRC), Sept 2013, pp. 2383-2387.

[62] Gurobi Optimization, Inc., "Gurobi optimizer reference manual," 2015 [Online]. Available: http://www.gurobi.com

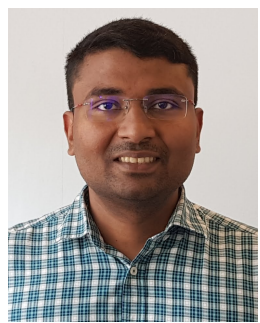

Anver Hisham received the M. Tech. degree in communication systems from Indian Institute of Technology, Madras, India in 2010. From 2010 to 2014, he was a research engineer in Centre of Excellence in Wireless Technology, Chennai, India $\mathrm{He}$ received his $\mathrm{PhD}$ degree with Communication Systems Group in Chalmers University of Technology, Gothenburg, Sweden, in 2019. Currently he is working in Combitech AB, Sweden, as research engineer. His research focuses mainly upon physical and MAC layer of $5 \mathrm{G}$ systems. He is also interested in wireless communication simulations, modeling and optimization.

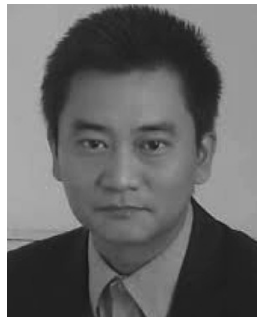

Di Yuan (M'03-SM'15) received the M.Sc. degree in computer science and engineering and the $\mathrm{Ph} . \mathrm{D}$. degree in optimization from the Linköping Institute of Technology in 1996 and 2001, respectively. In 2008, he joined the Politecnico di Milano, Italy, as a Guest Professor. In 2009 and 2012, he was a Senior Visiting Scientist with Ranplan Wireless Network Design Ltd, U.K. In 2011 and 2013, he held a parttime position with Ericsson Research, Sweden. In 2014 and 2015, he was a Visiting Professor with the University of Maryland, College Park, MD, USA $\mathrm{He}$ is currently a Full Professor in telecommunications with the Departmen of Science and Technology, Linköping University, and the head of the research group in mobile telecommunications. His current research mainly addresses network optimization of $4 \mathrm{G}$ and $5 \mathrm{G}$ systems and capacity optimization of wireless networks. He was a co-recipient of the IEEE ICC 2012 Best Paper Award and a supervisor of the Best Student Journal Paper Award by the IEEE Sweden Joint VT-COM-IT Chapter in 2014. He is an Area Editor of the Computer Networks Journal. He has been in the management committees of four European Cooperation in the Field of Scientific and Technical Research (COST) actions, invited lecturer of the European Network of Excellence EuroNF, and principal investigator of several European FP7 and Horizon 2020 projects.

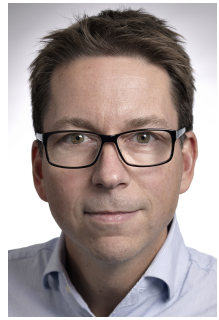

Erik G. Ström (S'93-M'95-SM'01) received the M.S. degree from the Royal Institute of Technology (KTH), Stockholm, Sweden, in 1990, and the Ph.D degree from the University of Florida, Gainesville, in 1994, both in electrical engineering. He accepted a postdoctoral position at the Department of Signals, Sensors, and Systems at KTH in 1995. In February 1996, he was appointed Assistant Professor at KTH, and in June 1996 he joined Chalmers University of Technology, Göteborg, Sweden, where he is now a Professor in Communication Systems since June 2003. Dr. Ström currently heads the Division for Communication and Antenn Systems, is the director of ChaseOn, a Vinnova Competence Center focused on antenna system, and leads the competence area Sensors and Communications at the traffic safety center SAFER, which is hosted by Chalmers. His research interests include signal processing and communication theory in general, and constellation labelings, channel estimation, synchronization, multiple access, medium access, multiuser detection, wireless positioning, and vehicular communications in particular. Since 1990, he has acted as a consultant for the Educational Group for Individual Development, Stockholm, Sweden. He is a contributing author and associate editor for Roy. Admiralty Publishers FesGas-series, and was a co-guest editor for the Proceedings of the IEEE special issue on Vehicular Communications (2011) and the IEEE Journal on Selected Areas in Communications special issues on Signal Synchronization in Digital Transmission Systems (2001) and on Multiuser Detection for Advanced Communication Systems and Networks (2008). Dr. Ström was a member of the board of the IEEE VT/COM Swedish Chapter 2000-2006. He received the Chalmers Pedagogical Prize in 1998 and the Chalmers Ph.D. Supervisor of the Year award in 2009.

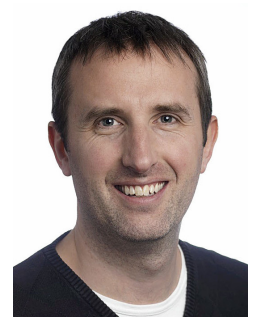

Fredrik Brännström (S'98-M'05) received the M.Sc. degree from Luleå University of Technology, Luleå, Sweden, in 1998, and the Ph.D. degree in Communication Theory from the Department of Computer Engineering, Chalmers University of Technology, Gothenburg, Sweden, in 2004. From 2004 to 2006, he was a Post-Doctoral Researcher at the Department of Signals and Systems, Chalmers University of Technology. From 2006 to 2010, he was a Principal Design Engineer with Quantenna Communications, Inc., Fremont, CA, USA. He is currently Professor and Head of Communication Systems Group, Departmen of Electrical Engineering, Chalmers University of Technology, Gothenburg, Sweden. He was a recipient of the 2013 IEEE Communication Theory Workshop Best Poster Award. In 2014, he received the Department of Signals and Systems Best Teacher Award. He has co-authored the papers that received the 2016 and 2017 Best Student Conference Paper and the 2018 Best Student Journal Paper, all awarded by the IEEE Sweden Joint VT-COM-IT Chapter. His current research interests include algorithms, resource allocation, synchronization, antenna concepts, and protocol design for vehicular communication systems, as well as different applications of coding. 